\title{
IMPROVING THE Ni I ATOMIC MODEL FOR SOLAR AND STELLAR ATMOSPHERIC MODELS
}

\author{
M. C. Vieytes ${ }^{1}$ AND J. M. Fontenla ${ }^{2}$ \\ ${ }^{1}$ Instituto de de Astronomía y Física del Espacio, CONICET and UNTREF, Buenos Aires, Argentina; mariela@iafe.uba.ar \\ ${ }^{2}$ North West Research Associates, 3380 Mitchell Lane, Boulder, CO 80301, USA; johnf@ digidyna.com \\ Received 2012 December 19; accepted 2013 March 13; published 2013 May 10
}

\begin{abstract}
Neutral nickel (Ni I) is abundant in the solar atmosphere and is one of the important elements that contribute to the emission and absorption of radiation in the spectral range between 1900 and $3900 \AA$. Previously, the Solar Radiation Physical Modeling (SRPM) models of the solar atmosphere only considered a few levels of this species. Here, we improve the Ni I atomic model by taking into account 61 levels and 490 spectral lines. We compute the populations of these levels in full NLTE using the SRPM code and compare the resulting emerging spectrum with observations. The present atomic model significantly improves the calculation of the solar spectral irradiance at near-UV wavelengths, which is important for Earth atmospheric studies, and particularly for ozone chemistry.
\end{abstract}

Key words: line: formation - radiative transfer - Sun: atmosphere - Sun: UV radiation

\section{INTRODUCTION}

The radiative flux spectrum incident at the top of Earth's atmosphere is known as the solar spectral irradiance (SSI). The near-UV range of the SSI plays a fundamental role in ozone chemistry. Most of Earth's atmospheric ozone is produced in the stratosphere as a result of photodissociation of molecular oxygen by UV solar radiation in the Schumann-Runge bands at wavelengths shorter than $\sim 1750 \AA$, and is destroyed by ozone photodissociation in the Hartley-Huggins bands (1900-3900 Å; Fontenla et al. 1999). A change in the amount of near-UV SSI directly affects the destruction of ozone, leading to tropospheric effects. Ozone changes also affect the amount of biologically harmful near-UV radiation reaching Earth's surface (Brasseur 1997). For these reasons, there is a need for an accurate calculation of the solar radiation that reaches the top of Earth's atmosphere in the near-UV range.

The Solar Radiation Physical Modeling (SRPM) system is a code library that permits one to compute the radiance (emergent intensity from the solar surface) spectrum of the Sun based on physical models of the solar atmosphere (see Fontenla et al. 2006, 2007, 2009). The code solves the radiative transfer and non-LTE statistical equilibrium assuming a specified one-dimensional plane-parallel geometry or a spherical symmetry depending on the study. The paper by Fontenla et al. (2011) applied the SRPM code and a set of physical models of various features observed on the solar surface to the calculation of high spectral-resolution SSI ranging from extreme-ultraviolet to far-infrared. The results obtained in that paper allow one to study the effects of SSI changes on Earth and other planetary atmospheres. Comparisons show a generally very good agreement between the observed and synthetic irradiance, but significant differences occur at some wavelengths.

Important discrepancies, especially in the near-UV range, reflect the need for more accurate and complete atomic models of some important species emitting and absorbing in that range. $\mathrm{Ni}$ I is an important species, considering its abundance in the solar atmosphere, and it has strong lines in the near-UV range. The present paper focuses on improving the calculation of the solar spectra in this range by improving the Ni I atomic model and using the SRPM system to compute the non-LTE populations and the emergent intensities. Comparisons are shown between the new results and observations of radiance and irradiance.
Only a few publications concerning the Ni I NLTE calculations are found in the literature, and most of them are about the formation of the Ni I $6769.64 \AA$ line. In helioseismology, this line is used for observing line-of-sight velocity (Doppler shift) by the Michelson Doppler Imager (MDI) on board Solar and Heliospheric Observatory $(\mathrm{SOHO})$ and by the ground-based Global Oscillations Network Group (GONG).

Bruls (1993) studied the Ni I 6769.64 A line using an atomic model with 19 energy levels. The atomic data used for the calculation were from Corliss \& Sugar (1981). The level populations and emergent intensities were computed in NLTE using the VAL C model by Vernazza et al. (1981). This paper confirmed that the line is a good choice for helioseismology.

More recently, Norton et al. (2006) compared the Ni I $6769.64 \AA$ and the Fe I $6173 \AA$ lines, both of which are candidates to be used by the Helioseismic and Magnetic Imager (HMI) on board the Solar Dynamics Observatory for observing Doppler velocities and magnetic fields. They adopted an Ni I atomic model with 25 energy levels and the same source of atomic data as in the previously mentioned work. The NLTE calculations were carried out again using model $\mathrm{C}$ from Vernazza et al. (1981) and also using sunspot umbra model M from Maltby et al. (1986). The HMI team selected the Fe I spectral line because of its better performance in magnetic diagnostics.

Considering the spectral lines and term system of $\mathrm{Ni}$, the results of Litzén et al. (1993) increased the number of known Ni I levels reported by Corliss \& Sugar (1981) from 185 to 286 , and the number of classified $\mathrm{Ni}$ I lines as expanded from 1071 to 1996 . This work is the source used by the National Institute of Standards and Technology (NIST) database version 4 (Ralchenko et al. 2011). The lowest 61 energy levels in the NIST tabulation are adopted in the present paper because they correspond to the most important lines, particularly in the near-UV.

In the present work, we calculate the quiet-Sun spectrum, as defined by the mix of solar features described by Fontenla et al. (2011), using the same set of physical models but improving the $\mathrm{Ni}$ I atomic model from the original 10 to the present 61 levels, and compare the computed spectrum with observations of several Ni I line profiles.

This paper is arranged as follows. The new Ni I atomic model is presented in Section 2. In Section 3, we show some results concerning the radiative transfer and NLTE calculations. In 


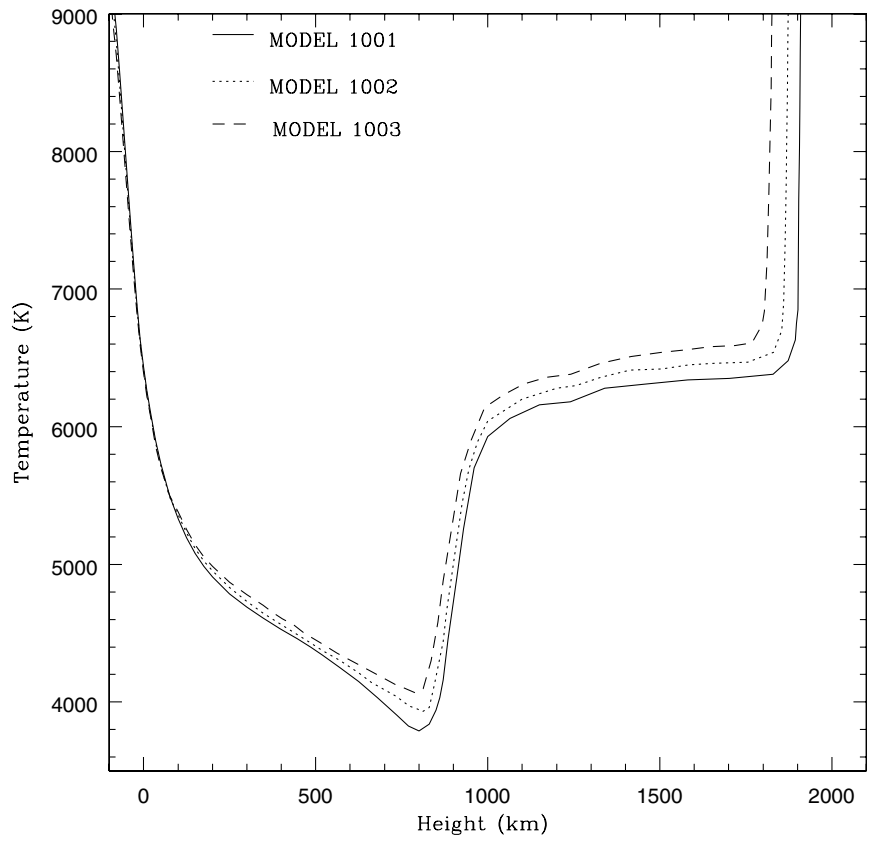

Figure 1. Atmospheric models for the solar features used to calculate the quietSun spectrum. These models were built by Fontenla et al. (2011).

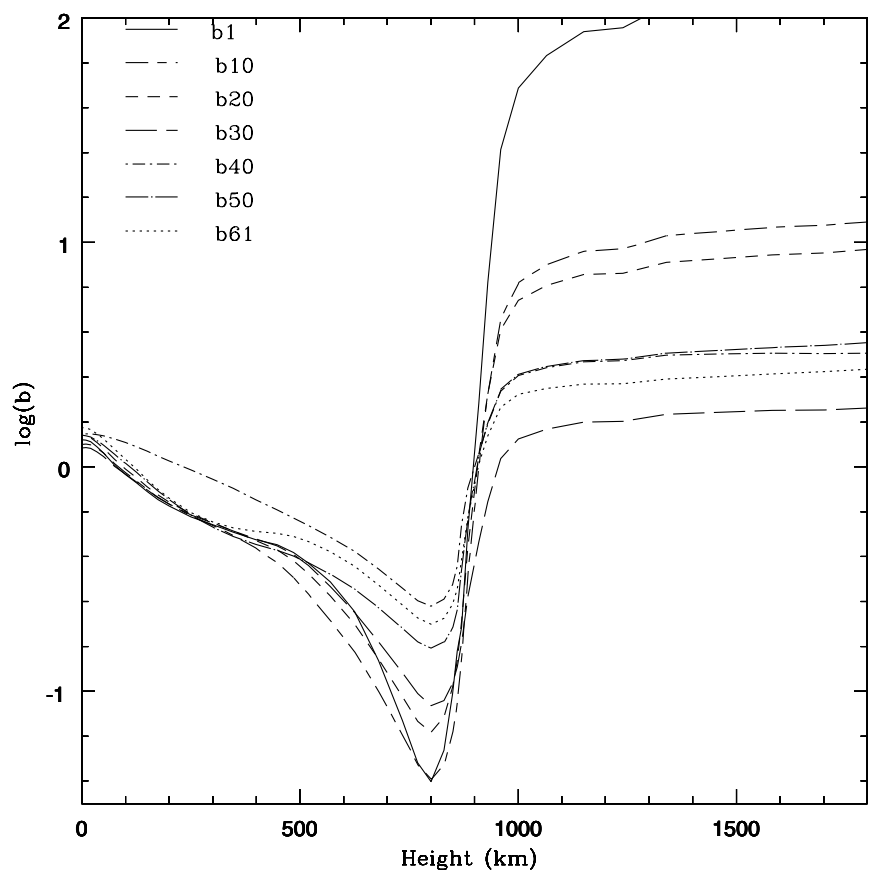

Figure 2. Departure coefficients $b$ for selected levels of Ni I in atmospheric model 1001.

Section 4, we compare the computed quiet-Sun spectra with observations. Finally, in Section 5, we discuss our results.

\section{THE IMPROVED Ni I ATOMIC MODEL}

The improvement of the $\mathrm{Ni}$ I atomic model is primarily motivated by the goal of producing a better computation and comparison with observations of the near-UV SSI.

Observations in this spectral range are scarce because atmospheric absorption, especially by ozone, makes ground observations impossible. SSI observations from space usually have limited spectral resolution.

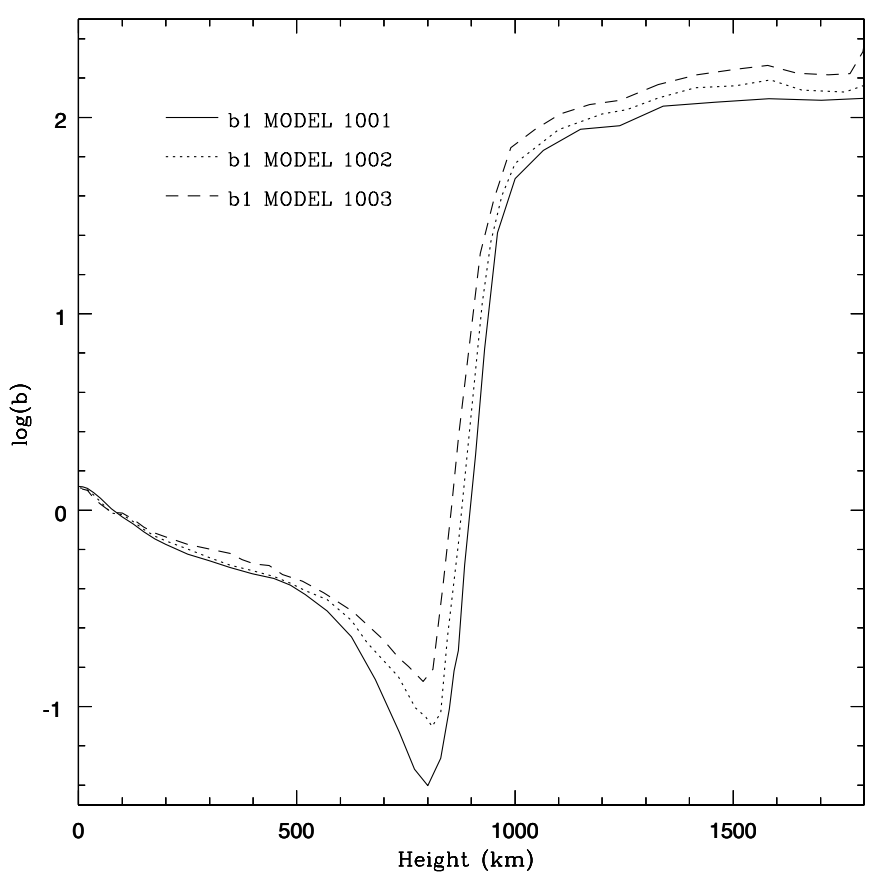

Figure 3. Departure coefficients $b 1$ for the ground state of Ni I in the atmospheric models used to calculate the solar spectrum.

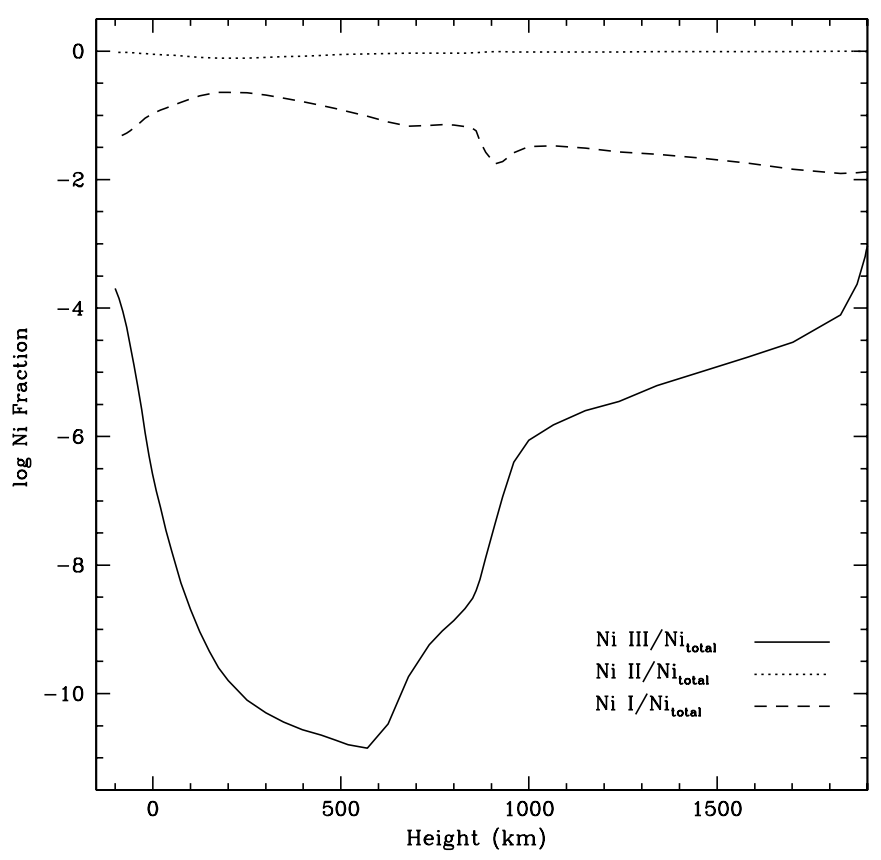

Figure 4. $\mathrm{Ni}$ I, Ni II, and $\mathrm{Ni}$ III densities relative to the $\mathrm{Ni}_{\text {total }}$ density for atmospheric model 1001 as a function of height.

A limitation to computing an accurate spectrum is the availability of accurate atomic data for NLTE calculations. The NIST atomic spectra database (Ralchenko et al. 2011) lists 503 $\mathrm{Ni}$ I spectral lines in the range $1900-7000 \AA$, with 266 belonging to the near-UV range (1900-3900 $\AA$ ).

To reproduce most of the strong Ni I lines in the near-UV range, it is necessary to consider an atomic model with 61 energy levels and, as described by Ralchenko et al. (2011), the corresponding sublevels defined by the $J$ quantum number. Using this number of levels, only 34 of the NIST-listed lines are not included. 

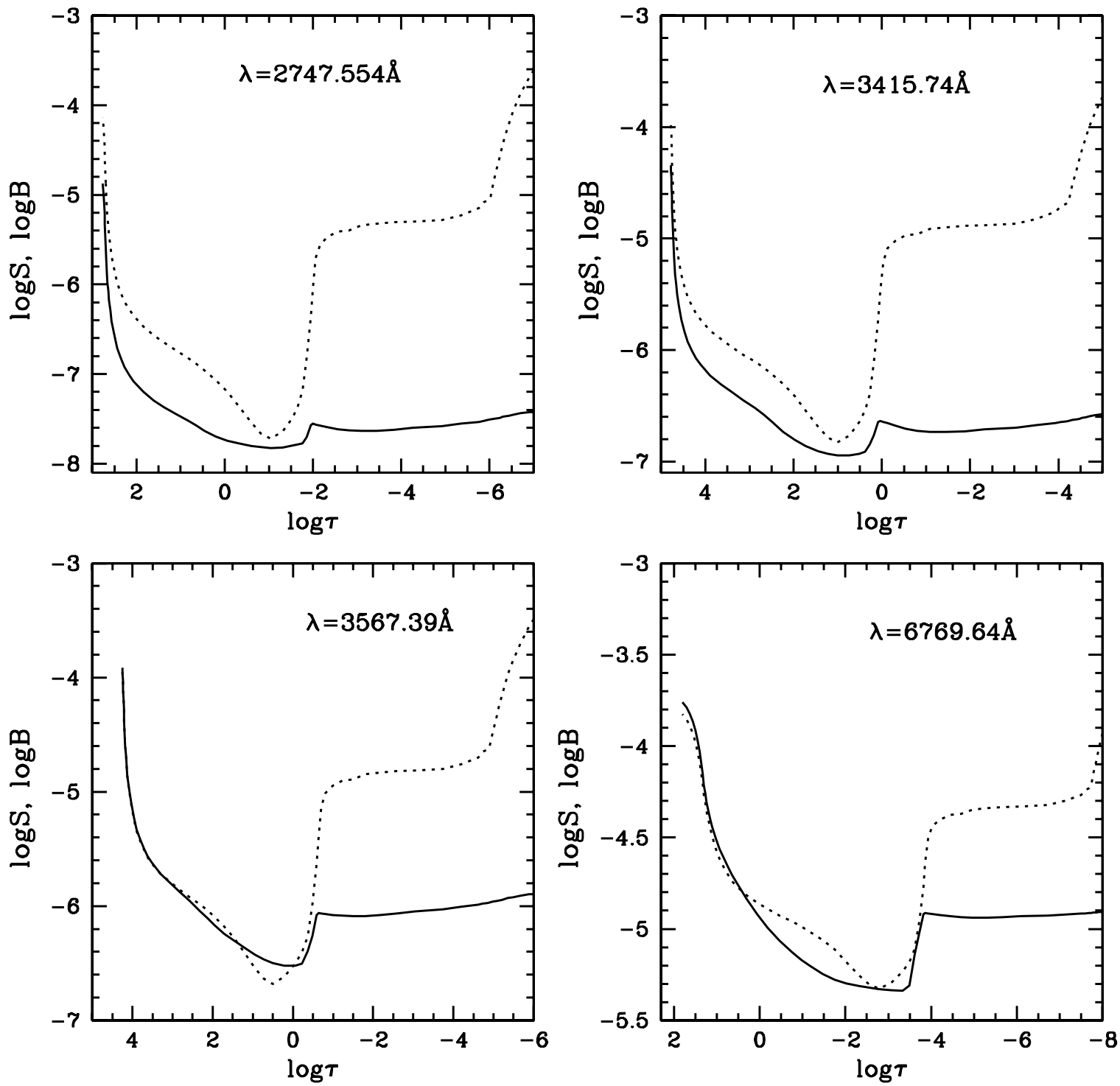

Figure 5. Line source function ( $S$; solid line) and Planck function ( $B$; doted line) as a function of the optical depth at line center, $\tau$, for selected spectral lines.

The level energies, line wavelengths, and weighted oscillator strength $g f$ values were taken from the NIST atomic spectra database. The collisional strength parameters were obtained from the Seaton (1962) approximation, and the photoionization cross sections were estimated from similar atomic structure species. The reason for these approximations is the absence of better Ni I atomic data in the literature. For the radiative, Stark, and Van der Waals broadening parameters, we used the values estimated by Kurucz (1994).

In Table 1, we present a description of the 61 atomic levels and sublevels of energy considered. The first column lists the configuration, and the second column the associated term. The third column shows the values of the $J$ quantum number that define the sublevels, and the fourth column presents the sublevel energies. The fifth column lists the level number in our scheme.

\section{THE NLTE CALCULATIONS}

We carried out the calculations using the latest semiempirical models of the solar atmosphere described by Fontenla et al. (2011). The quiet-Sun spectrum was built by weighted averaging of the three models listed in Table 2 and shown in Figure 1. To reproduce the low solar activity state during the period 2008-2009, we considered $80 \%$ of the solar disk to be covered by an internetwork feature (model 1001, B), 19\% by a network feature (model 1002, D), and $1 \%$ by an active network (model 1003, F).

The populations for the atomic species listed in Table 2 of Fontenla et al. (2011) were all calculated in full NLTE as in Fontenla et al. (2011), but now with the new Ni I atomic model that increased the number of energy levels from 10 to 61 .

We calculated the LTE departure coefficients ( $b$ values), defined by the ratio of the computed level populations relative to those resulting from the Saha-Boltzmann distribution, to the next ion stage (Menzel definition; Rutten 2003). Figure 2 shows the calculated $b$ 's for many levels that resulted from atmospheric model 1001.

The general behavior is similar to that described in Fontenla et al. (2009). At heights above $\sim 900 \mathrm{~km}$ (i.e., above the temperature minimum region), the $b$ values are greater than unity, with the largest value corresponding to the ground level. The opposite occurs in the low chromosphere and the 

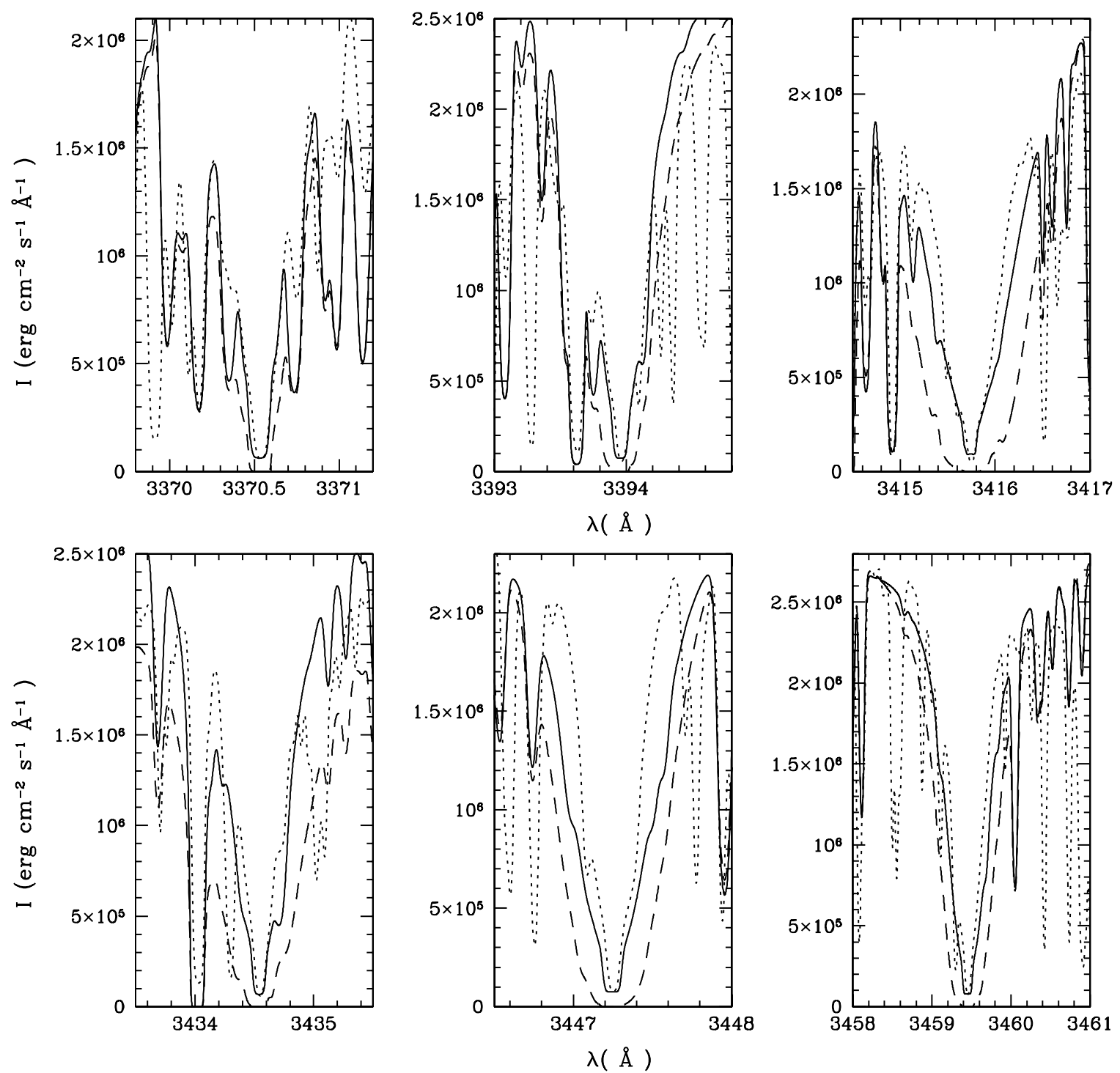

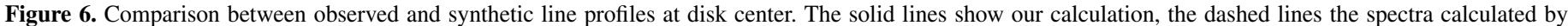

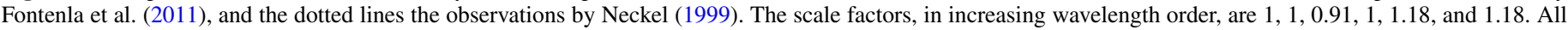
wavelengths are in a vacuum.

temperature minimum region where the $b$ values are smaller than unity. In general, as the energy of the level increases, the departure coefficients are closer to unity, but fluctuations occur depending on how the various levels are connected by permitted transitions.

In Figure 3 , we plot the $b_{1}$ departure coefficients of the ground level for the three models used to reproduce the quiet-Sun spectrum. Although the general behavior is similar, the region where $b_{1}$ is smaller than unity becomes thinner, and closer to unity, as the minimum temperature of the atmosphere increases.

In Figure 4, we show the ion density fractions of $\mathrm{Ni} / \mathrm{Ni}_{\text {total }}, \mathrm{Ni}$ II $/ \mathrm{Ni}_{\text {total }}$, and $\mathrm{Ni}$ III $/ \mathrm{Ni}_{\text {total }}$ with height. Throughout the atmosphere, the total Ni II density is nearly the total $\mathrm{Ni}$ density. Above $\sim 300 \mathrm{~km}, \mathrm{Ni} / \mathrm{Ni}_{\text {total }}$ drops slowly. Above $\sim 600 \mathrm{~km}, \mathrm{Ni}$ III $/ \mathrm{Ni}_{\text {total }}$ begins to increase, although below the chromosphere-corona transition region it remains several orders of magnitude lower than the other two density fractions.

In Figure 5, we plot the line source function (solid line) and the local Planck function (doted line) versus the optical depth at the line center $\tau$ for four important line transitions. In a first approximation, the line center forms at $\tau=1$ for the disk center and $\tau=2 / 3$ for the irradiance. Considering this plot, departures from LTE produce a large difference between these functions for most of the plotted $\tau$ values, and specially around $\tau=1$. The heights at which $\tau=1$ are as follows: $\sim 597 \mathrm{~km}$ for the $2747.554 \AA$ line, $\sim 945 \mathrm{~km}$ for the $3415.74 \AA$ line, $\sim 840 \mathrm{~km}$ for the $3567.39 \AA$ line, and $\sim 323 \mathrm{~km}$ for the $6769.64 \AA$ line. Thus, the centers of these lines form in the low chromospheric layers at and below the temperature region where the NLTE effects are strong, and their source functions cannot be well described by any simple approximation.

\section{COMPARISON OF THE CALCULATED SPECTRA WITH OBSERVATIONS}

We carried out our calculations of the solar spectrum with the new Ni I atomic model, using the latest semiempirical models of the solar atmosphere described by Fontenla et al. (2011). The quiet-Sun spectrum was constructed by weighted averaging of the spectra from the three models listed in Table 2 and shown 

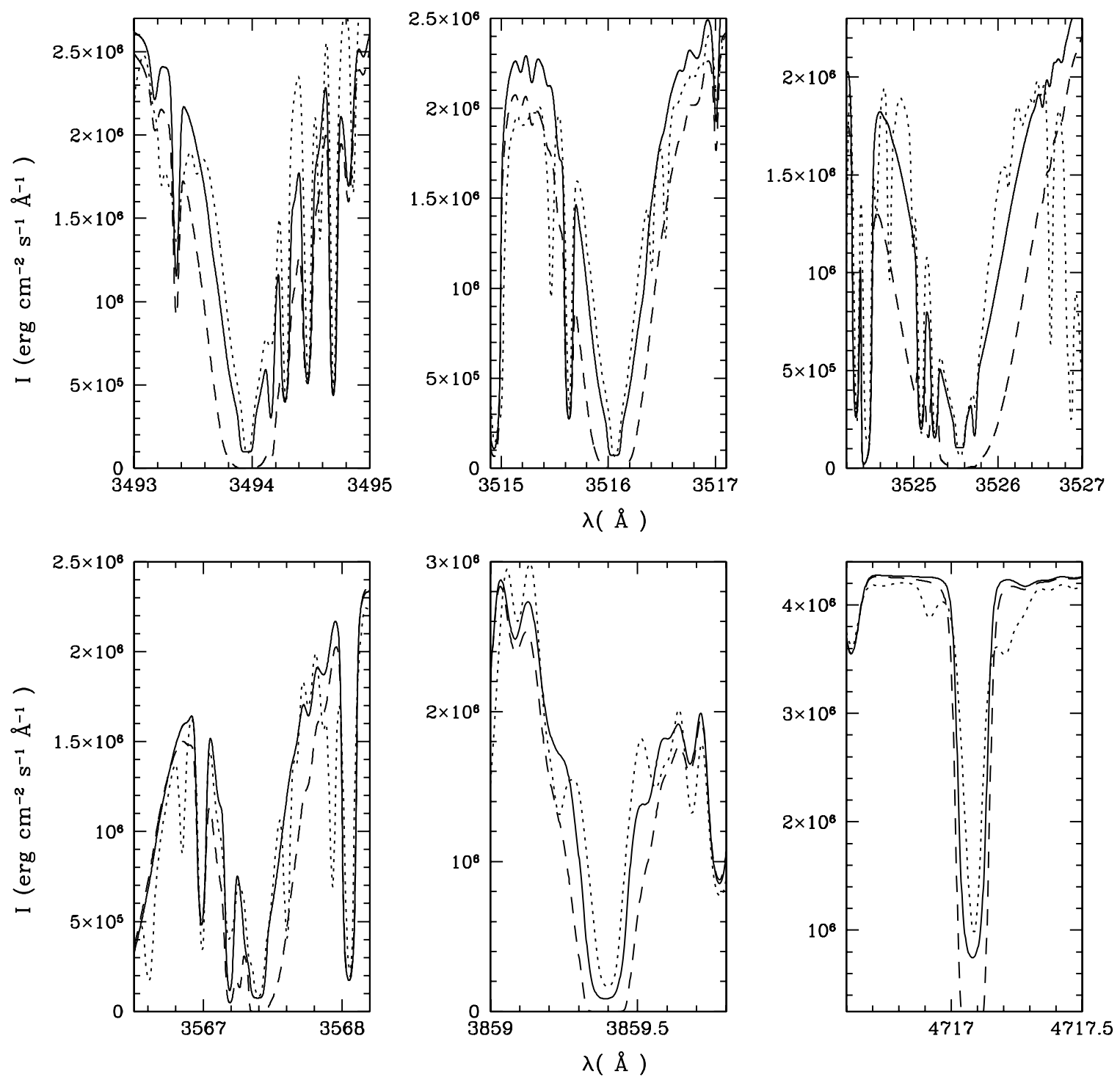

Figure 7. Comparison between observed and synthetic line profiles at disk center. The solid lines show our calculation, the dashed lines the spectra calculated by Fontenla et al. (2011), and the dotted lines the Neckel (1999) solar atlas. The scale factors, in increasing wavelength order, are 1.28, 1, 0.91, 1, 1.18, and 1.25. All wavelengths are in a vacuum.

in Figure 1. To reproduce the low solar activity state during the period 2008-2009, the solar disk is considered to be covered by the relative areas of the features indicated in Table 2.

The information about the Ni l lines and energy levels that we compare with observations are presented in Table 3. The last column in this table indicates the figure number where this transition is plotted. Many of the line profiles shown in the figures correspond to transitions between energy levels that were never before computed in full NLTE.

The computed spectral lines in the range from 3290 to $7000 \AA$ are compared with the observations at disk center in the Fourier transform spectrometer (FTS) solar atlas by Neckel (1999). The absolute intensities of these observations were from the Kitt Peak FTS data as described by Neckel \& Labs (1984a, 1984b) and Neckel (1994). The absolute calibration of this disk-center spectrum is not very reliable, as explained by Neckel (1999). For this reason, we scaled the observations to match the continuum near the line profiles with the calculations. Figures 6-8 show the comparison of several line profiles and the scaling factors.
The last panel in Figure 7 shows the Ni I $6769.64 \AA$ (vacuum wavelength) line used by the $\mathrm{SOHO} / \mathrm{MDI}$ and GONG instruments.

The differences between the two computed profiles plotted in these figures are due to the different number of levels in both NLTE calculations that was mentioned before. Several Ni I lines were absent in the spectra calculated by Fontenla et al. (2011), while others are too deep (e.g., dashed trace in Figure 7). The latter are improved in our present calculation by the inclusion of many more levels.

In some cases, the calculated line profile is wider than the observed one. We also recomputed the line profiles using the broadening parameters included in the Vienna Atomic Line Database (Kupka et al. 2000), but the results obtained were similar.

Comparisons with observations were also carried out with near-UV irradiance spectrum in the range from 190 to $310 \mathrm{~nm}$. The data from space instruments by Thuillier et al. (2003) and by Harder et al. (2010) have a reliable absolute calibration. However, these data have low spectral resolution. In Figure 9, we 

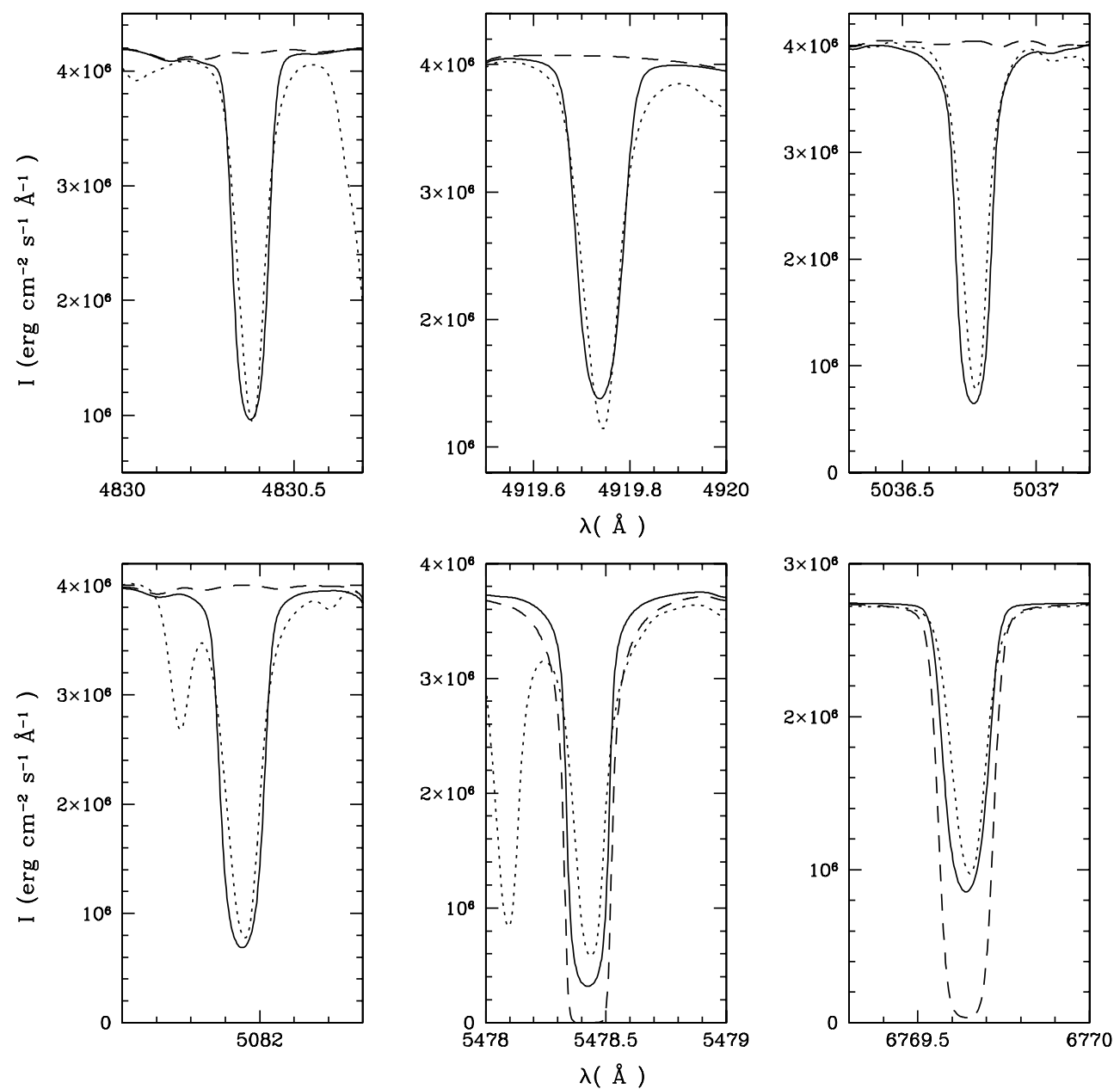

Figure 8. Comparison between observed and synthetic line profiles at disk center. The solid lines show our calculation, the dashed lines the spectra calculated by Fontenla et al. (2011), and the dotted lines the Neckel (1999) solar atlas. The scale factor for all the lines is 1.28. All wavelengths are in a vacuum.

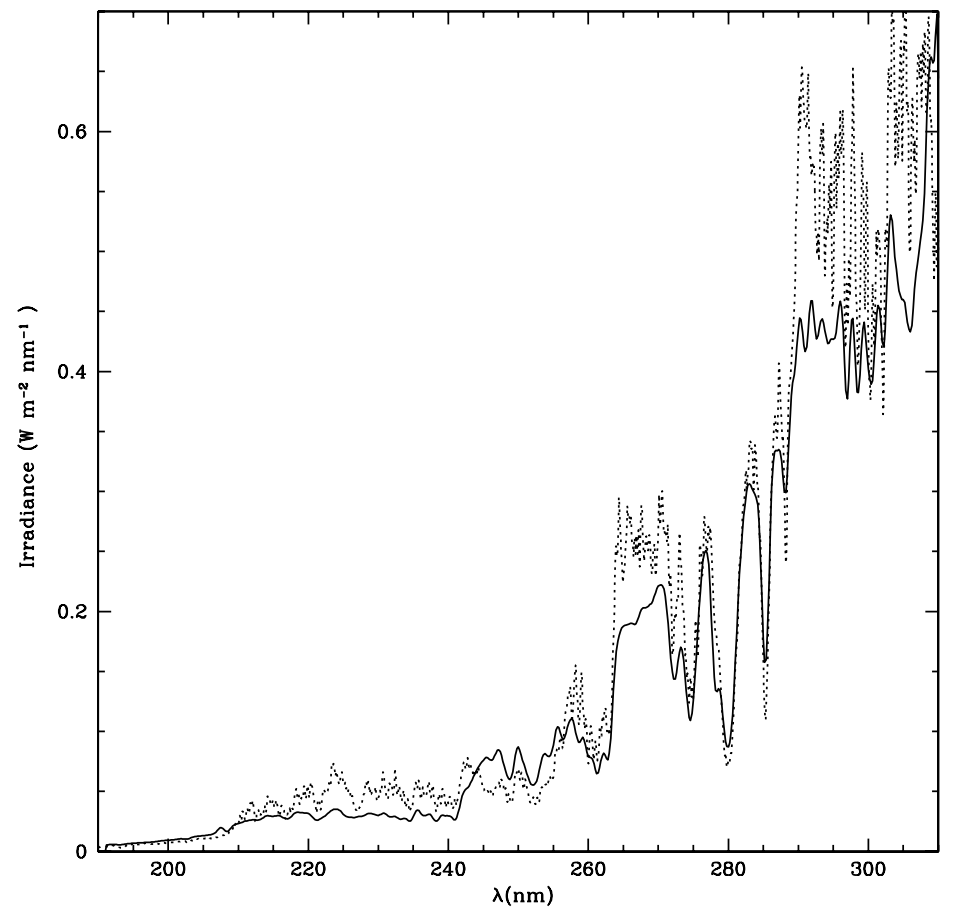

Figure 9. Comparison between observed and synthetic spectral irradiance between 190 and $310 \mathrm{~nm}$. The solid lines show our calculation, and the dashed lines the Composite 3 reference spectrum derived by Thuillier et al. (2003). 

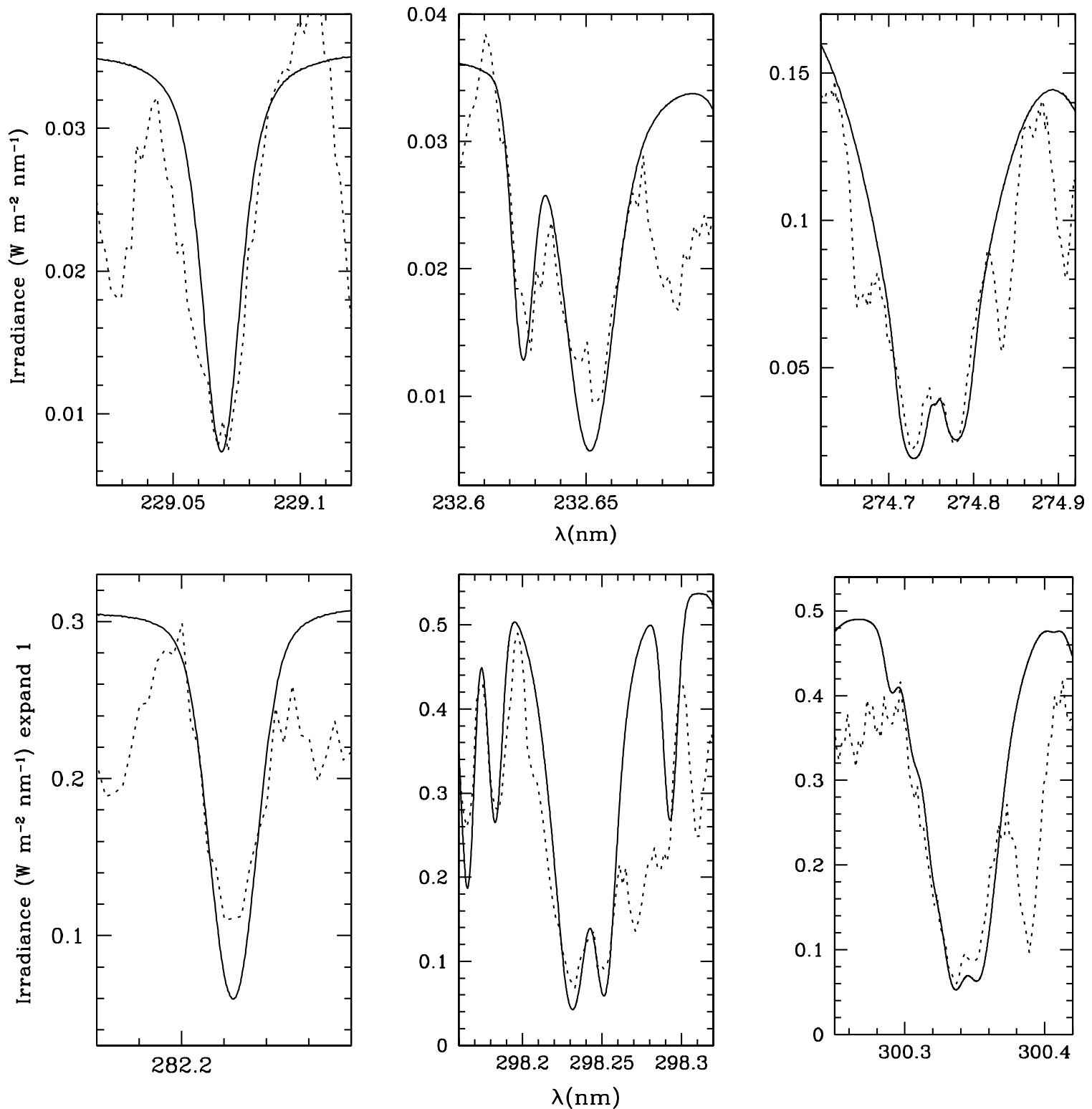

Figure 10. Comparison between observed and synthetic irradiance line profiles. The solid lines show our calculation, and the dotted lines the H\&A observations. All wavelengths are in a vacuum. The scale factors between the observed and the calculated profile are, in increasing wavelength order, 1.9, 3.3, 3.5, 1.8, 0.75, and 0.83 .

show the comparison between our computed spectral irradiance and the Composite 3 reference spectrum by Thuillier et al. (2003). To simulate the resolution of the observations, the calculated spectral irradiance was convolved with a $1 \mathrm{~nm}$ FWHM $\cos ^{2}$ function as in Fontenla et al. (2011).

The observations of the irradiance spectrum by Hall \& Anderson (1991) and Anderson \& Hall (1989), hereafter H\&A, were obtained from stratospheric balloon flights that are very difficult to calibrate because of the atmospheric absorption. However, these observations have a fairly good spectral resolution of $0.01 \mathrm{~nm}$. For the present comparison, the H\&A spectrum was scaled to match overall the values in the calculated spectrum. In Figure 10, we compare several computed Ni I line profiles with the scaled H\&A irradiance observations. The comparison shows good agreement between calculations and observations.

\section{SUMMARY}

This work improves the Ni I atomic model to include the majority of the lines in the near UV range that are listed in the NIST atomic database. Several of these lines are very strong and have not been calculated in full NLTE by previous papers.

The results shown here display fairly good agreement with the observed Ni I lines because we include full NLTE calculations for an atomic model with 61 levels, which have fine structure sublevels. This agreement does not require any changes of the Fontenla et al. (2011) atmospheric models.

Also, we reproduce well the Ni I $6769.64 \AA$ line used in the MDI and GONG observations, allowing a better understanding of the line formation in different solar features, and investigating the impact of solar activity on its behavior. 
Table 1

The First 61 Levels of Ni I Used in the Present Paper (Ralchenko et al. 2011)

\begin{tabular}{|c|c|c|c|c|}
\hline Configuration & Term & $J$ & $\begin{array}{c}\text { Level } \\
\left(\mathrm{cm}^{-1}\right)\end{array}$ & Level Number \\
\hline \multirow[t]{3}{*}{$3 d^{8}\left({ }^{3} F\right) 4 s^{2}$} & ${ }^{3} F$ & 4 & 0.000 & 1 \\
\hline & & 3 & 1332.164 & \\
\hline & & 2 & 2216.550 & \\
\hline \multirow[t]{3}{*}{$3 d^{9}\left({ }^{2} D\right) 4 s$} & ${ }^{3} D$ & 3 & 204.787 & 2 \\
\hline & & 2 & 879.816 & \\
\hline & & 1 & 1713.087 & \\
\hline $3 d^{9}\left({ }^{2} D\right) 4 s$ & ${ }^{1} D$ & 2 & 3409.937 & 3 \\
\hline $3 d^{8}\left({ }^{1} D\right) 4 s^{2}$ & ${ }^{1} D$ & 2 & 13521.347 & 4 \\
\hline $3 d 10$ & ${ }^{1} S$ & 0 & 14728.840 & 5 \\
\hline \multirow[t]{3}{*}{$3 d^{8}\left({ }^{3} P\right) 4 s^{2}$} & ${ }^{3} P$ & 2 & 15609.844 & 6 \\
\hline & & 1 & 15734.001 & \\
\hline & & 0 & 16017.306 & \\
\hline $3 d^{8}\left({ }^{1} G\right) 4 s^{2}$ & ${ }^{1} G$ & 4 & 22102.325 & 7 \\
\hline \multirow[t]{5}{*}{$3 d^{8}\left({ }^{3} F\right) 4 s 4 p\left({ }^{3} P^{o}\right)$} & ${ }^{3} D^{o}$ & 4 & 25753.553 & 8 \\
\hline & & 3 & 26665.887 & \\
\hline & & 2 & 27414.868 & \\
\hline & & 1 & 27943.524 & \\
\hline & & 0 & 28212.998 & \\
\hline \multirow[t]{5}{*}{$3 d^{8}\left({ }^{3} F\right) 4 s 4 p\left({ }^{3} P^{o}\right)$} & ${ }^{5} G^{o}$ & 6 & 27260.894 & 9 \\
\hline & & 5 & 27580.391 & \\
\hline & & 4 & 28068.065 & \\
\hline & & 3 & 28578.018 & \\
\hline & & 2 & 29013.206 & \\
\hline \multirow[t]{5}{*}{$3 d^{8}\left({ }^{3} F\right) 4 s 4 p\left({ }^{3} P^{o}\right)$} & ${ }^{5} F^{o}$ & 5 & 28542.105 & 10 \\
\hline & & 4 & 29084.456 & \\
\hline & & 3 & 29832.779 & \\
\hline & & 2 & 30163.124 & \\
\hline & & 1 & 30392.003 & \\
\hline \multirow[t]{3}{*}{$3 d^{9}\left({ }^{2} D\right) 4 p$} & ${ }^{3} P^{o}$ & 2 & 28569.203 & 11 \\
\hline & & 1 & 29500.674 & \\
\hline & & 0 & 30192.251 & \\
\hline \multirow{3}{*}{$3 d^{9}\left({ }^{2} D\right) 4 p$} & ${ }^{3} F^{o}$ & 4 & 29480.989 & 12 \\
\hline & & 3 & 29320.762 & \\
\hline & & 2 & 30619.414 & \\
\hline \multirow[t]{3}{*}{$3 d^{9}\left({ }^{2} D\right) 4 p$} & ${ }^{3} D^{o}$ & 3 & 29668.893 & 13 \\
\hline & & 2 & 29888.477 & \\
\hline & & 1 & 30912.817 & \\
\hline $3 d^{9}\left({ }^{2} D\right) 4 p$ & & 3 & 29668.918 & 14 \\
\hline \multirow[t]{3}{*}{$3 d^{8}\left({ }^{3} F\right) 4 s 4 p\left({ }^{3} P^{o}\right)$} & ${ }^{3} G^{o}$ & 5 & 30922.734 & 15 \\
\hline & & 4 & 30979.749 & \\
\hline & & 3 & 31786.162 & \\
\hline $3 d^{9}\left({ }^{2} D\right) 4 p$ & ${ }^{1} F^{o}$ & 3 & 31031.020 & 16 \\
\hline $3 d^{9}\left({ }^{2} D\right) 4 p$ & ${ }^{1} D^{o}$ & 2 & 31441.635 & 17 \\
\hline \multirow[t]{3}{*}{$3 d^{8}\left({ }^{3} F\right) 4 s 4 p\left({ }^{3} P^{o}\right)$} & ${ }^{3} F^{o}$ & 4 & 32973.376 & 18 \\
\hline & & 3 & 33112.334 & \\
\hline & & 2 & 34163.264 & \\
\hline $3 d^{9}\left({ }^{2} D\right) 4 p$ & ${ }^{1} P^{o}$ & 1 & 32982.260 & 19 \\
\hline \multirow[t]{3}{*}{$3 d^{8}\left({ }^{3} F\right) 4 s 4 p\left({ }^{3} P^{o}\right)$} & ${ }^{3} D^{o}$ & 3 & 33500.822 & 20 \\
\hline & & 2 & 33610.890 & \\
\hline & & 1 & 34408.555 & \\
\hline $3 d^{8}\left({ }^{3} F\right) 4 s 4 p\left({ }^{3} P^{o}\right)$ & ${ }^{1} G^{o}$ & 4 & 33590.130 & 21 \\
\hline $3 d^{8}\left({ }^{3} F\right) 4 s 4 p\left({ }^{3} P^{o}\right)$ & ${ }^{1} F^{o}$ & 3 & 35639.122 & 22 \\
\hline $3 d^{8}\left({ }^{3} F\right) 4 s 4 p\left({ }^{3} P^{o}\right)$ & ${ }^{1} D^{o}$ & 2 & 36600.791 & 23 \\
\hline \multirow[t]{3}{*}{$3 d^{8}\left({ }^{3} P\right) 4 s 4 p\left({ }^{3} P\right)$} & ${ }^{5} P^{o}$ & 3 & 40361.249 & 24 \\
\hline & & 2 & 40484.212 & \\
\hline & & 1 & 40768.996 & \\
\hline \multirow[t]{3}{*}{$3 d^{8}\left({ }^{1} D\right) 4 s 4 p\left({ }^{3} P^{o}\right)$} & ${ }^{3} F^{o}$ & 4 & 42585.212 & 25 \\
\hline & & 3 & 42767.853 & \\
\hline & & 2 & 42954.203 & \\
\hline $3 d^{9}\left({ }^{2} D_{5 / 2}\right) 5 s$ & ${ }^{2}[5 / 2]$ & 3 & 42605.945 & 26 \\
\hline & & 2 & 42790.010 & \\
\hline $3 d^{8}\left({ }^{1} D\right) 4 s 4 p\left({ }^{3} P^{o}\right)$ & ${ }^{3} D^{o}$ & 3 & 42620.994 & 27 \\
\hline & & 2 & 42653.661 & \\
\hline & & 1 & 42656.289 & \\
\hline
\end{tabular}

Table 1

(Continued)

\begin{tabular}{|c|c|c|c|c|}
\hline Configuration & Term & $J$ & $\begin{array}{c}\text { Level } \\
\left(\mathrm{cm}^{-1}\right)\end{array}$ & Level Number \\
\hline \multirow[t]{3}{*}{$3 d^{8}\left({ }^{3} F\right) 4 s 4 p\left({ }^{1} P^{o}\right)$} & ${ }^{3} G^{o}$ & 5 & 43089.578 & 28 \\
\hline & & 4 & 44314.904 & \\
\hline & & 3 & 44565.037 & \\
\hline \multirow[t]{3}{*}{$3 d^{8}\left({ }^{3} F\right) 4 s 4 p\left({ }^{1} P^{o}\right)$} & ${ }^{3} F^{o}$ & 4 & 43258.726 & 29 \\
\hline & & 3 & 45281.089 & \\
\hline & & 2 & 45418.804 & \\
\hline \multirow[t]{2}{*}{$3 d^{8}\left({ }^{1} D\right) 4 s 4 p\left({ }^{3} P^{o}\right)$} & ${ }^{3} P^{o}$ & 1 & 43463.981 & 30 \\
\hline & & 2 & 43933.408 & \\
\hline \multirow[t]{3}{*}{$3 d^{8}\left({ }^{3} F\right) 4 s 4 p\left({ }^{1} P^{o}\right)$} & ${ }^{3} D^{o}$ & 3 & 43654.903 & 31 \\
\hline & & 2 & 44475.099 & \\
\hline & & 1 & 45122.383 & \\
\hline $3 d^{8}\left({ }^{3} F\right) 4 s 4 p\left({ }^{1} P^{o}\right)$ & & 3 & 43654.974 & 32 \\
\hline \multirow[t]{5}{*}{$3 d^{8}\left({ }^{3} P\right) 4 s 4 p\left({ }^{3} P^{o}\right)$} & ${ }^{5} D^{o}$ & 4 & 44540.525 & 33 \\
\hline & & 3 & 44206.099 & \\
\hline & & 2 & 44093.773 & \\
\hline & & 1 & 44132.250 & \\
\hline & & 0 & 44414.955 & \\
\hline \multirow[t]{2}{*}{$3 d^{9}\left({ }^{2} D_{3 / 2}\right) 5 s$} & ${ }^{2}[3 / 2]$ & 1 & 44112.173 & 34 \\
\hline & & 2 & 44262.599 & \\
\hline $3 d^{8}\left({ }^{3} P\right) 4 s 4 p\left({ }^{3} P^{o}\right)$ & & 4 & 44336.10 & 35 \\
\hline \multirow[t]{3}{*}{$3 d^{8}\left({ }^{3} P\right) 4 s 4 p\left({ }^{3} P^{o}\right)$} & ${ }^{3} P^{o}$ & 2 & 46522.866 & 36 \\
\hline & & 1 & 47208.149 & \\
\hline & & 0 & 47686.587 & \\
\hline \multirow[t]{3}{*}{$3 d^{8}\left({ }^{3} P\right) 4 s 4 p\left({ }^{3} P^{o}\right)$} & ${ }^{3} D^{o}$ & 3 & 47030.102 & 37 \\
\hline & & 2 & 47139.337 & \\
\hline & & 1 & 47424.785 & \\
\hline $3 d^{8}\left({ }^{3} P\right) 4 s 4 p\left({ }^{3} P^{o}\right)$ & ${ }^{5} S$ & 2 & 47328.784 & 38 \\
\hline \multirow[t]{5}{*}{$3 d^{8} 4 s\left({ }^{4} F\right) 5 s$} & ${ }^{5} F$ & 5 & 48466.490 & 39 \\
\hline & & 4 & 49085.982 & \\
\hline & & 3 & 49777.569 & \\
\hline & & 2 & 50346.427 & \\
\hline & & 1 & 50744.552 & \\
\hline $3 d^{9}\left({ }^{2} D\right) 5 p$ & ${ }^{1} F^{o}$ & 3 & 48671.049 & 40 \\
\hline \multirow[t]{3}{*}{$3 d^{9}\left({ }^{2} D\right) 5 p$} & ${ }^{3} F^{o}$ & 4 & 48715.586 & 41 \\
\hline & & 3 & 50142.991 & \\
\hline & & 2 & 50039.191 & \\
\hline \multirow[t]{3}{*}{$3 d^{9}\left({ }^{2} D\right) 5 p$} & ${ }^{3} P^{o}$ & 2 & 48735.290 & 42 \\
\hline & & 1 & 49196.181 & \\
\hline & & 0 & 50138.458 & \\
\hline $3 d^{8}\left({ }^{3} P\right) 4 s 4 p\left({ }^{3} P^{o}\right)$ & ${ }^{1} P^{o}$ & 1 & 48818.097 & 43 \\
\hline \multirow[t]{2}{*}{$3 d^{9}\left({ }^{2} D_{5 / 2}\right) 4 d$} & ${ }^{2}[1 / 2]$ & 1 & 48953.316 & 44 \\
\hline & & 0 & 49610.345 & \\
\hline $3 d^{9}\left({ }^{2} D\right) 5 p$ & ${ }^{1} D^{o}$ & 2 & 49032.926 & 45 \\
\hline \multirow[t]{2}{*}{$3 d^{9}\left({ }^{2} D_{5 / 2}\right) 4 d$} & ${ }^{2}[9 / 2]$ & 5 & 49158.480 & 46 \\
\hline & & 4 & 49174.770 & \\
\hline \multirow{2}{*}{$3 d^{9}\left({ }^{2} D_{5 / 2}\right) 4 d$} & ${ }^{2}[3 / 2]$ & 2 & 49159.030 & 47 \\
\hline & & 1 & 49171.151 & \\
\hline $3 d^{8}\left({ }^{3} P\right) 4 s 4 p\left({ }^{3} P^{o}\right)$ & ${ }^{1} D^{o}$ & 2 & 49185.138 & 48 \\
\hline $3 d^{9}\left({ }^{2} D_{5 / 2}\right) 4 d$ & ${ }^{2}[5 / 2]$ & 3 & 49271.540 & 49 \\
\hline & & 2 & 49327.811 & \\
\hline $3 d^{9}\left({ }^{2} D_{5 / 2}\right) 4 d$ & ${ }^{2}[7 / 2]$ & 3 & 49313.814 & 50 \\
\hline & & 4 & 49332.593 & \\
\hline $3 d^{9}\left({ }^{2} D\right) 5 p$ & ${ }^{3} D^{o}$ & 3 & 49328.140 & 51 \\
\hline & & 2 & 50689.489 & \\
\hline & & 1 & 50851.199 & \\
\hline $3 d^{8}\left({ }^{3} P\right) 4 s 4 p\left({ }^{3} P^{o}\right)$ & ${ }^{3} S^{o}$ & 1 & 49403.386 & 52 \\
\hline $3 d^{9}\left({ }^{2} D\right) 5 p$ & & 3 & 50142.8 & 53 \\
\hline $3 d^{8}\left({ }^{1} \mathrm{~S}\right) 4 s^{2}$ & ${ }^{1} \mathrm{~S}$ & 0 & 50276.321 & 54 \\
\hline $3 d^{9}\left({ }^{2} D\right) 5 p$ & ${ }^{1} P^{o}$ & 1 & 50458.192 & 55 \\
\hline $3 d^{8} 4 s\left({ }^{4} F\right) 5 s$ & ${ }^{3} F$ & 4 & 50466.131 & 56 \\
\hline & & 3 & 51306.038 & \\
\hline & & 2 & 52040.523 & \\
\hline $3 d^{9}\left({ }^{2} D_{3 / 2}\right) 4 d$ & ${ }^{2}[1 / 2]$ & 1 & 50536.703 & 57 \\
\hline & & 0 & 51457.250 & \\
\hline
\end{tabular}


Table 1

(Continued)

\begin{tabular}{lcccc}
\hline \hline Configuration & Term & $J$ & $\begin{array}{c}\text { Level } \\
\left(\mathrm{cm}^{-1}\right)\end{array}$ & Level Number \\
\hline $3 d^{9}\left({ }^{2} D_{3 / 2}\right) 4 d$ & ${ }^{2}[7 / 2]$ & 3 & 50677.555 & 58 \\
$3 d^{9}\left({ }^{2} D_{3 / 2}\right) 4 d$ & $2[3 / 2]$ & 1 & 50716.896 & 59 \\
$3 d^{8}\left({ }^{1} \mathrm{G}\right) 4 s 4 p\left({ }^{3} P^{o}\right)$ & ${ }^{3} F^{o}$ & 4 & 50789.303 & 60 \\
& & 3 & 51124.662 & \\
$3 d^{9}\left({ }^{2} D_{3 / 2}\right) 4 d$ & 2 & 51343.547 & 61 \\
& $2[5 / 2]$ & 3 & 50832.001 & \\
& & 2 & 50834.401 & \\
\hline
\end{tabular}

Table 2

Quiet-Sun Feature Components and Their Respective Models

\begin{tabular}{|c|c|c|c|}
\hline \multirow[t]{2}{*}{ Feature } & \multirow[t]{2}{*}{ Description } & \multirow{2}{*}{\multicolumn{2}{|c|}{$\begin{array}{c}\text { Photosphere-Chromosphere Relative Area on } \\
\text { Model Index }\end{array}$}} \\
\hline & & & \\
\hline B & Quiet-Sun internetwork & 1001 & $80 \%$ \\
\hline $\mathrm{D}$ & Quiet-Sun network lane & 1002 & $19 \%$ \\
\hline $\mathrm{F}$ & Enhanced network & 1003 & $1 \%$ \\
\hline
\end{tabular}

Note. We follow the feature and model index designations as presented in Fontenla et al. (2011).

Table 3

Transition Lines Plotted in Figures 6, 7, 8, and 10

\begin{tabular}{|c|c|c|c|}
\hline $\begin{array}{l}\lambda_{\text {vac }} \\
(\AA)\end{array}$ & $\begin{array}{l}\text { Upper Level } \\
\left(\mathrm{cm}^{-1}\right)\end{array}$ & $\begin{array}{c}\text { Lower Level } \\
\left(\mathrm{cm}^{-1}\right)\end{array}$ & Figure Number \\
\hline 2296.69 & 43654.974 & 0 & 10 \\
\hline 2326.515 & 44314.904 & 1332.164 & 10 \\
\hline 2747.554 & 36600.791 & 204.787 & 10 \\
\hline 2822.122 & 35639.122 & 204.787 & 10 \\
\hline 2982.516 & 34408.555 & 879.816 & 10 \\
\hline 3003.36 & 33500.822 & 204.787 & 10 \\
\hline 3370.531 & 29668.918 & 0 & 6 \\
\hline 3393.957 & 29668.918 & 204.787 & 6 \\
\hline 3415.744 & 29480.989 & 204.787 & 6 \\
\hline 3434.54 & 29320.762 & 204.787 & 6 \\
\hline 3447.247 & 29888.477 & 879.816 & 6 \\
\hline 3459.451 & 30619.414 & 1713.087 & 6 \\
\hline 3493.956 & 29500.674 & 879.816 & 7 \\
\hline 3516.057 & 29320.762 & 879.816 & 7 \\
\hline 3525.544 & 28569.203 & 204.787 & 7 \\
\hline 3567.39 & 31441.635 & 3409.937 & 7 \\
\hline 3859.391 & 29320.762 & 3409.937 & 7 \\
\hline 4717.081 & 49777.569 & 28578.018 & 7 \\
\hline 4830.372 & 49271.540 & 28569.203 & 8 \\
\hline 4919.737 & 51306.038 & 30979.749 & 8 \\
\hline 5036.766 & 49174.770 & 29320.762 & 8 \\
\hline 5081.949 & 49158.480 & 29480.989 & 8 \\
\hline
\end{tabular}

Table 3

(Continued)

\begin{tabular}{lccc}
\hline \hline $\begin{array}{l}\lambda_{\text {vac }} \\
(\AA)\end{array}$ & $\begin{array}{c}\text { Upper Level } \\
\left(\mathrm{cm}^{-1}\right)\end{array}$ & $\begin{array}{c}\text { Lower Level } \\
\left(\mathrm{cm}^{-1}\right)\end{array}$ & Figure Number \\
\hline 5478.426 & 32982.260 & 14728.84 & 8 \\
6769.64 & 29500.674 & 14728.84 & 8 \\
\hline
\end{tabular}

Notes. Column 1: the vacuum wavelength. Columns 2 and 3: the energy value for the upper and lower sublevel, respectively, involved in the transition (Ralchenko et al. 2011).

The results presented in this work stress the importance of full NLTE calculation and the use of a sufficient number of levels for a realistic computation of line profiles. From these results, it is clear that using the Fontenla et al. (2011) models for spectra calculations with LTE codes will not properly compute the deep Ni I lines and likely others as well. This is because the calculations here show that the line source function substantially departs from LTE in the low chromosphere and at the temperature minimum. LTE values will systematically produce wrong results with the Fontenla et al. (2011) models.

M.C.V. acknowledges support from PICT 0746-2010 ANPCyT, and Programación Científica 2010-2011 UNTREF grants. J.M.F. was supported by LWS-NASA grant NNX09AJ22G.

\section{REFERENCES}

Anderson, G., \& Hall, L. 1989, JGR, 94, D56435

Brasseur, G. (ed.) 1997, NATO ASI Series: The Stratosphere and Its Role in the Climate System, Vol. 54 (Berlin: Springer-Verlag)

Bruls, J. 1993, A\&A, 269, 509

Corliss, C., \& Sugar, J. 1981, JPCRD, 10, 197

Fontenla, J., Curdt, W., Haberreiter, M., Harder, J., \& Tian, H. 2009, ApJ, 707,482

Fontenla, J., Harder, J., Livingston, W., Snow, M., \& Woods, T. 2011, JGR, 116, D20108

Fontenla, J., White, O., Fox, P., Avrett, E., \& Kurucz, R. 1999, ApJ, 518, 480

Fontenla, J. M., Avrett, E., Thuillier, G., \& Harder, J. 2006, ApJ, 639, 441

Fontenla, J. M., Balasubramaniam, K. S., \& Harder, J. 2007, ApJ, 667, 1243

Hall, L., \& Anderson, G. 1991, JGR, 96, D12927

Harder, J., Thuillier, G., Richard, E., et al. 2010, SoPh, 263, 3

Kupka, F., Ryabchikova, T. A., Piskunov, N. E., Stempels, H. C., \& Weiss, W. W. 2000, BaltA, 9, 590

Kurucz, R. L. 1994, Kurucz CD-ROM No. 20-22 (Cambridge, MA: SAO)

Litzén, U., Brault, J., \& Thorne, A. 1993, PhyS, 47, 628

Maltby, P., Avrett, E., Carlsson, M., et al. 1986, ApJ, 306, 284

Neckel, H. 1994, in IAU Colloq. 143, The Sun as a Variable Star: Solar and Stellar Irradiance Variations, ed. J. M. Pap, C. Frolich, H. S. Hudson, \& S Solanki (Cambridge: Cambridge Univ. Press), 37

Neckel, H. 1999, SoPh, 184, 421

Neckel, H., \& Labs, D. 1984a, SoPh, 90, 205

Neckel, H., \& Labs, D. 1984b, SoPh, 92, 391

Norton, A. A., Graham, J. P., Ulrich, R. K., et al. 2006, SoPh, 239, 69

Ralchenko, Yu., Kramida, A. E., Reader, J., \& NIST ASD Team 2011, NIST Atomic Spectra Database (ver. 4.1.0), Online, http://physics.nist.gov/asd (Gaithersburg, MD: National Institute of Standards and Technology)

Rutten, R. J. 2003, Radiative Transfer in Stellar Atmospheres (8th ed.; Utrecht Sterrekundig Instuut Ultrecht)

Seaton, J. M. 1962, PPS, 79, 1105

Thuillier, G., Herse, M., Labs, D., et al. 2003, SoPh, 214, 1

Vernazza, J., Avrett, E., \& Loeser, R. 1981, ApJS, 45, 635 